\title{
Plasma sarcosine does not distinguish early and advanced stages of prostate cancer
}

\author{
L Bohm, A M Serafin, P Fernandez, G Van der Watt, P J D Bouic, J Harvey
}

Introduction. Diagnosis of prostate cancer by prostate specific antigen (PSA) is error-prone and cannot distinguish benign prostatic hyperplasia (BPH) from malignant disease, nor identify aggressive and indolent types.

Methods. We determined serum sarcosine (N-methylglycine) in 328 cancer patients by gas chromatography (GC)/mass spectroscopy (MS) and searched for correlations with early (stage T1/T2) and advanced (stage T3/T4) disease.

Results. Serum sarcosine of male control patients ranged from 1.7 $\mu \mathrm{mol} / \mathrm{l}$ to $4.8 \mu \mathrm{mol} / \mathrm{l}$. In prostate cancer patients, sarcosine ranged from $2.8 \mu \mathrm{mol} / \mathrm{l}$ to $20.1 \mu \mathrm{mol} / \mathrm{l}$. Expressed as the sarcosine/alanine ratio, serum control values were $9.4 \pm 5.5 \times 10^{-3}$ (mean $\pm \mathrm{SD}$ ) compared with $21.6 \pm 9.0 ; 28.5 \pm 16.6 ; 22.7 \pm 7.7$ and $22.2 \pm 11.0$ for patients diagnosed with T1, T2, T3 and T4 prostate tumours, respectively. The small differences between T1, T2, T3 and T4 patients were not statistically significant $(p=0.51)$. However, the conventional PSA marker significantly correlated with $\mathrm{T}$ stage in these patients $(r=0.63 ; p<0.009)$.

Conclusions. The median sarcosine/alanine ratios among patients with early and advanced prostatic cancer ranged from 21.6 \pm 9.0 to $28.5 \pm 16.6$ and were fairly constant, showing no statistically significant differences between T-stages. The results are consistent with published data in urine and serum which find differences between controls and patients with metastatic prostate cancer to be small and sarcosine to be uninformative regarding prostate cancer progression. By multi-comparison of PSA with T-stages in the same group of patients, we found significant correlations confirming the well-known merits and limitations of this marker.

S Afr Med J 2012;102(8):677-679. DOI:10.7196/SAMJ.5768
The identification of prostate cancer remains problematic; no single, simple procedure exists for a reliable diagnosis. Prostate specific antigen (PSA), while non-invasive and easily measurable in serum, is not specific owing to false-positives from benign prostatic hyperplasia $(\mathrm{BPH})$, inflammatory conditions and prostatic trauma. Additional diagnostic information must therefore be obtained by transrectal ultrasonography (TRUS) and digital rectal examination (DRE) to assess prostate size and morphology. Final confirmation of a malignancy requires histopathological analysis of several biopsies. A remaining hurdle in the identification process is the existence of indolent organ-confined disease and aggressive metastatic prostate cancer that can only be distinguished by additional imaging or nuclear medicine procedures.

In a new approach to this diagnostic dilemma, it has been argued that screening for changes in metabolite expression resulting from

Department of Medical Imaging and Clinical Oncology, Faculty of Health Sciences, Stellenbosch University

L Bohm, PhD

A M Serafin, MTech, $\mathrm{PhD}$

Department of Urology, Faculty of Health Sciences, Stellenbosch University P Fernandez, $\mathrm{PhD}$

Department of Chemical Pathology, University of Cape Town and Red Cross War Memorial Children's Hospital, Cape Town

G Van der Watt, MB ChB, MMed, FCPath SA, DA SA

Department of Pathology, Faculty of Health Sciences, Stellenbosch University P J D Bouic, $\mathrm{PhD}$

Department of Mathematical Statistics, Stellenbosch University J Harvey, $\mathrm{PhD}$ gene silencing and gene activation could be used to identify a specific biological marker that increases in the transformation process. Therefore, a paper elaborating on metabolite expression in clinical samples of benign, localised and metastatic prostate cancer $^{1}$ was enthusiastically received and debated in editorials..$^{2-5}$ The proposal that sarcosine ( $N$-methylglycine) (a metabolite of choline found in urine) may be characteristic for prostate cancer progression has since been examined in other laboratories. ${ }^{1}$ These investigations found sarcosine levels in urine ${ }^{6}$ and serum $^{7}$ to be constant, irrespective of sample pathology. From our own and published preliminary measurements, it is nevertheless clear that sarcosine-alanine ratios in prostate tumour patients are often elevated above controls and are spread over a wider range. ${ }^{1,7,17} \mathrm{~A}$ relationship between sarcosine and disease therefore cannot as yet be totally ruled out. Interest in sarcosine as a marker molecule remains topical, as shown by a recent modification of sarcosine assay by GC/MS in urine. ${ }^{8}$

We present measurements in plasma of prostate cancer patients characterised by PSA, tumour stage and Gleason score using GC/MS for sarcosine methodology $y^{9,10}$ to elaborate further on the suitability of sarcosine as a marker for prostate cancer.

\section{Methods}

Patient blood was collected by venipuncture and centrifuged, and clear supernatants were stored at $-20^{\circ} \mathrm{C}$. The AxSYM total PSA was determined by a MEIA microparticle enzyme immune assay (Abbott) and expressed as $\mathrm{ng} / \mathrm{ml}$. Ethical approval was granted by the University of Stellenbosch Health Research Ethics Committee (N09/11/330).

Sarcosine and alanine were quantified in plasma by gaschromatograph mass spectrometry. Briefly, amino acids were extracted from $100 \mu \mathrm{l}$ of acidified plasma by solid phase extraction onto a cation exchange column before being washed, eluted and derivatised to their corresponding alkyl chloroformates using a commercial kit (EZ:faast, Phenomenex, Torrance CA, USA). After derivatisation, the amino acids were extracted into solvent and $2 \mu \mathrm{l}$ injected into an Agilent 7890A/5975C GCMS system fitted with a Zebron ZB AAA $10 \mathrm{~m}$ x $0.25 \mathrm{~mm}$ capillary GC column. The sample 
was injected at a $1: 10$ split and $250^{\circ} \mathrm{C}$. Helium was used as the carrier gas at $1.65 \mathrm{ml} / \mathrm{min}$, and the column heated from $110^{\circ} \mathrm{C}$ to $320^{\circ} \mathrm{C}$ at $30^{\circ} \mathrm{C}$ per minute. The amino acids were quantified using a norvaline internal standard against standard curves generated from traceable calibrators using the following ions and (qualifier ions): alanine $-\mathrm{mz}$ 130 , sarcosine $-\mathrm{mz} 217$, and norvaline $-\mathrm{mz} 158$. Fig. 1 demonstrates a total ion chromatogram obtained from an equimolar $20 \mu \mathrm{mol} / \mathrm{l}$ solution of amino acids. Abundance of alanine in plasma was used as the internal reference, and all results were expressed as the sarcosine/ alanine ratio, as previously suggested. ${ }^{1}$

\section{Results}

Absolute sarcosine levels in serum/plasma of male control patients were found to be in the range of $1.7-4.8 \mu \mathrm{mol} / \mathrm{l}$ while alanine used as the internal standard was in the range of $301-553 \mu \mathrm{mol} / \mathrm{l}$. To circumvent variations owing to diet and metabolic factors, sarcosine is now commonly expressed as the sarcosine/alanine ratio. The median sarcosine/alanine ratios in tumour patients were fairly constant and in the range of $15-25 \times 10^{-3}$. Within a given tumour category, e.g. T2, sarcosine/alanine ratios can vary by factors as high as 10 , while the median sarcosine/alanine ratios in early and advanced tumours remain in a fairly narrow range of $15-25 \times 10^{-3}$ with a trend towards lower values in the T3/T4 group of tumors (Fig. 2). The sarcosine results were obtained in plasma of patients who had undergone transurethral resection (TURP) to alleviate urinary obstruction. Of these patients, $27 \%$ had benign prostatic hyperplasias $(\mathrm{BPH})$ and were not entered for sarcosine analysis. Samples entered for sarcosine determination fell into 2 groups: tumour stages T1 and T2, Gleason score <7; and advanced tumours T3 and T4 with a Gleason score $>7$. Fig. 2 shows sarcosine/alanine ratios in the $\mathrm{T} 1 / \mathrm{T} 2$ group of patients to be higher than in the T3/T4 group of

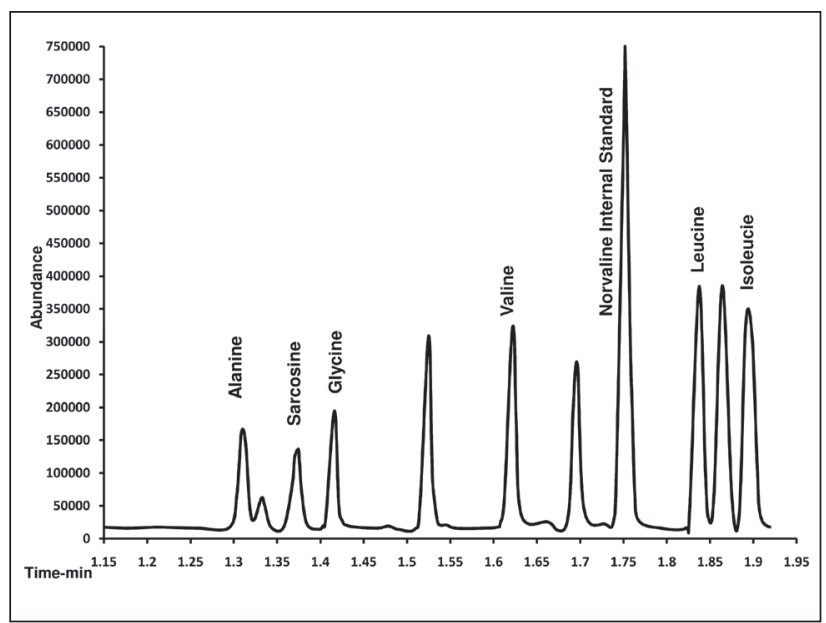

Fig. 1. Total GC/MS ion chromatogram of a $20 \mu \mathrm{mol} / \mathrm{l}$ solution of amino acids demonstrating the separation in plasma. $\mathrm{N}$-methylglycine (sarcosine) retention time is 1.38 minutes. Peak at 1.53 is $\alpha$-amino-butyric acid.

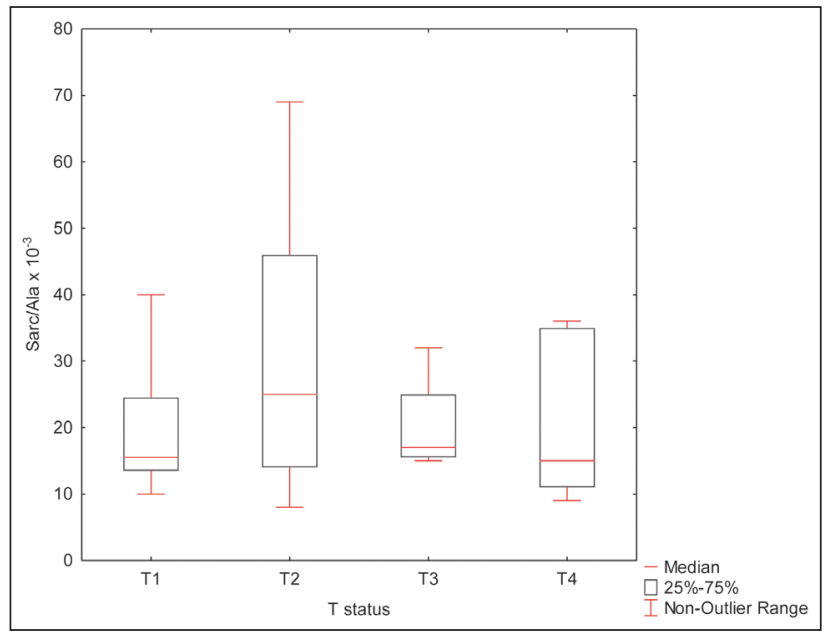

Fig. 2. Box plots of sarcosine/alanine ratios against T-status. Data were subjected to Kruskal-Wallis test T1 $(\mathrm{N}=20), T 2(\mathrm{~N}=17), T 3(\mathrm{~N}=11)$ and T4 $(\mathrm{N}=10)$. Differences between medians were not statistically significant $(\mathrm{p}=0.51)$.

patients. Two-tailed multiple comparison of sarcosine/alanine ratios with T-stages by Kruskal-Wallis multiple comparison test dialogue indicated that the differences between medians were not statistically different $(p=0.51)$.

Examining the relationship between tumour stage and PSA in a larger contingent of patients ( $N=325)$, we found PSA to be well below $200 \mathrm{ng} / \mathrm{ml}$ for the T1 and T2 group of patients, reaching $1200 \mathrm{ng} / \mathrm{ml}$ in the T3 and T4 group of patients. PSA and T stage were significantly correlated $(r=0.63$ and $p<0.009)$. Data for percentage free PSA were not available in our patients. By Kruskal-Wallis multiple comparison, we found that the PSA in T1 patients differed significantly from T3 and T4, and T2 differed from T4, while PSA did not separate T2 from $\mathrm{T} 3$ and $\mathrm{T} 1$ from T2 (Table 1, Fig. 3).

\section{Discussion}

Using urine samples, Jentzmik et al. found the sarcosine/creatinine ratios in patients with prostatectomy-based Gleason score $<7$ and Gleason score $>7$ not to be statistically different. ${ }^{6}$ Since sarcosine in urine is due to renal excretion, urine may not be a representative body fluid. ${ }^{6}$ Therefore, plasma may be a better source of abnormal metabolite expression. Following this reasoning, Struys et al. determined sarcosine in plasma from controls, localised prostate cancer, and metastatic castration-resistant prostate cancer. In this study, the mean sarcosine concentration was found to be at the level of $2.0 \mu \mathrm{mol} / \mathrm{l}$ with no statistical differences between the 3 sample categories. ${ }^{7}$ Our results (Fig. 2) are consistent with the findings on urine $^{6}$ and serum ${ }^{7}$ and show that, where prostate cancer is manifest, the sarcosine/alanine ratio cannot distinguish early and advanced disease. Specific alterations of sarcosine metabolism exist in folate

Table 1. Kruskal-Wallis multiple comparisons of PSA per T-stage, showing individual $p$-values*

\begin{tabular}{lllll}
\hline PSA & T1 & T2 & T3 & T4 \\
\hline T1 & & 0.173478 & 0.005280 & 0.000002 \\
T2 & 0.173478 & & 1.000000 & 0.010383 \\
T3 & 0.005280 & 1.000000 & 0.523807 \\
T4 & 0.000002 & 0.010383 & 0.523807 & \\
*See Fig. 3. & & & &
\end{tabular}




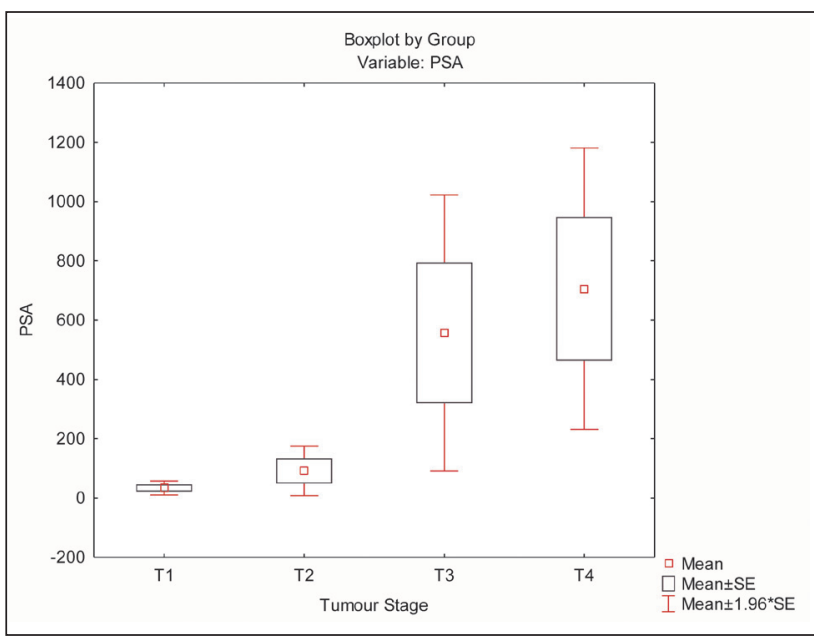

Fig. 3. Box plot of PSA against T-stage. PSA separates T1/T2 tumours from T3/T4 tumours. T1 $\mathrm{N}=89, T 2 \mathrm{~N}=80, T 3 \mathrm{~N}=78$, and T4 $\mathrm{N}=78$. By KruskalWallis test, T1 was found to be statistically different from T3 and T4; T2 was found to be statistically different from T4 only, and T3 was statistically different from $T 1$.

and in N-methylglycine-transferase deficiency where sarcosine reaches levels as high as $49.3 \mu \mathrm{mol} / \mathrm{l}^{13}$

Jentzmik et al. pointed out in their analysis of urine sediments ${ }^{6}$ that the sarcosine/creatinine ratios were insensitive to rectal palpation, in contrast with Sreekumar's original findings ${ }^{1}$ that observed such sensitivity. That sarcosine correlates with invasion and performs better than PSA to distinguish diagnostic classes in the grey zone of 2 - $10 \mathrm{ng} / \mathrm{ml} \mathrm{PSA}^{1}$ also could not be corroborated. ${ }^{6}$ The controversy about sarcosine as a new diagnostic marker has been debated in an editorial ${ }^{14}$ and several letters. ${ }^{15-18}$

A consistent observation in our analysis is that the sarcosinealanine ratios within patient groups can vary as much as 10 times, e.g. in T2 patients (Fig. 2). The median T1, T2, T3 and T4 ratios were found to be in the range of $15-25 \times 10^{-3}$ and to be fairly constant, showing no statistically significant differences. Publications on urine sediments showed sarcosine-alanine ratios to be higher than in serum and to vary from $70 \times 10^{-3}$ in controls to $100 \times 10^{-3}$ in prostate cancer patients, and a $2-3$ times variation of ratios within groups. ${ }^{17}$ From our data, we conclude that sarcosine has little merit as a biomarker and can give no useful additional information on prostate cancer progression. Also not supported is proof of the concept of sarcosine as a potential biomarker put forward in the exchange of views on sarcosine. ${ }^{17}$

The failure of sarcosine as a marker for prostate cancer prompted us to re-examine the diagnostic merits of PSA, using data from our teaching hospital at Tygerberg. PSA separates the T1 - T2 from the T3 - T4 group of tumours rather well (Fig. 3, Table 1). These results are in agreement with extensive PSA data showing that mean and median PSA values indeed differ between T-stages where they serve as a valuable indicators in the management of the disease. ${ }^{19,20}$ Our results add to the growing realisation that sarcosine as a potential biomarker of prostate cancer progression may not live up to expectations.

Acknowledgements. We thank Dr John Stanfliet for standard addition analyses, and Proff C F Heyns and A Ellmann for comments. A seeding grant from the Faculty of Health Sciences, Stellenbosch University, is also gratefully acknowledged.

\section{References}

1. Sreekumar A, Poisson LM, Rajendiran TM, et al. Metabolomic profiles delineate potential role for sarcosine in prostate cancer progression. Nature 2009;457(7231):910-914. [http://dx.doi.org/10.1038/ nature 07762

2. Pavlou M, Diamandis EP. The search for new prostate cancer biomarkers continues. Clinical Chemistry 2009.55(7);1277-1279. [http://dx.doi.org/ 10.1373/clinchem.2009.126870]

3. Kuehn BM. Promising marker found for deadly prostate cancer. JAMA 2009;301:1008. [http://dx.doi org/10.1001/jama.2009.299

4. Abate-Shen C, Shen MM. Diagnostics: the prostate cancer metabolome. Nature 2009;457:799-800. [http://dx.doi.org/10.1038/457799a]

5. Couzin J. Metabolite in urine may point to high-risk prostate cancer. Science 2009;323:865. [http:// dx.doi.org/10.1126/science.323.5916.865a]

6. Jentzmik F, Stephan C, Miller K, Schrader M, Erbersdobler A, et al. Sarcosine in urine after digital rectal examination fails as a marker in prostate cancer detection and identification of aggressive tumours. Eur Urol 2010;58:12-18. [http://dx.doi.org/10.1016/j.eururo.2010.01.035]

7. Struys EA, Heijboer AC, Van Moorselaar J, Jakobs C, Blankenstein MA. Serum sarcosine is not a marker for prostate cancer. Annals of Clin Biochem 2010;47:282. [http://dx.doi.org/10.1258/acb.2010.009270]

8. Cavaliere B, Macchione B, Monteleone M, Naccarato A Sindona G, Tagarelli A. Sarcosine as a marker in proste B, Macchione B, Monteleone M, Naccarato A, Sindona G, Tagarelli A. Sarcosine as a marke in prostate cancer progression: a rapid and simple method for its quantification in human urine by solid-phase microextraction-gas chromatography-triple quadrupole mass spectrometry. Anal Bioanal
Chem 2011;400(9):2903-2912. [http://dx.doi.org/10.1007/s00216-011-4960-0]

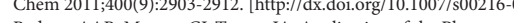

Badawy AAB, Morgan CJ, Turner JA. Application of the Phenomenex EZ:faast amino acid analysis kit for rapid gas-chromatographic determination of concentrations of plasma tryptophan and its brain uptake competitors. Amino Acids 2008;34:587-596. [http://dx.doi.org/10.1007/s00726-007-0012-7]

10. Kaspar H, Dettmer K, Gronwald W, Oefner PJ. Automated GC-MS analysis of free amino acids in biological fluids. J Chromatography 2008;870:222-232. [http://dx.doi.org/10.1016/j. jchromb.2008.06.018]

11. Allen RH, Stabler SP, Lindenbaum J. Serum betaine, $N, N$-dimethylglycine and $N$-methylglycine level in patients with cobalamin and folate deficiency and related inborn errors of metabolism. Metabolism 1993;42(11):1448-1460. [http://dx.doi.org/10.1016/0026-0495(93)90198-W]

12. Oon SF, Pennington SR, Fitzpatrick JM, Watson RWG. Biomarker research in prostate cancer towards utility, not futility. Nat Rev Urol 2011;8:131-138. [http://dx.doi.org/10.1038/nrurol.2011.11]

3. Mudd SH, Cerone R, Schiaffino MC, et al. Glycine N-methyltransferase deficiency: a novel inborn error causing persistent isolated hypermethioninaemia. J Inherit Metab Dis 2001:24(4):448-464. error causing persistent isolated hypermethio
[http://dx.doi.org/10.1023/A:1010577512912]

14. Schalken JA. Is urinary sarcosine useful to identify patients with significant prostate cancer? The trials and tribulations of biomarker development. Eur Urol 2010;58,19-21. [http://dx.doi.org/10.1016/j. eururo.2010.02.025]

15. Jentzmik F, Stephan C, Miller K, et al. Sarcosine in urine after digital rectal examination fails as a marker in prostate cancer detection and identification of aggressive tumours. Eur Urol 2010;58:12-18. [http://dx.doi.org/10.1016/j.eururo.2010.01.035]

16. Stephan C, Jentzmik F, Jung K. Reply: Is urinary sarcosine useful to identify patients with significan prostate cancer? The trials and tribulations of biomarker development. Eur Urol 2010;58:20-21. [http:// dx.doi.org/10.1016/j.eururo.2010.03.004]

17. Sreekumar A, Poisson LM, Rajendiran TM, et al. Reply: Sarcosine in urine after digital examination fails as a marker in prostate cancer detection and identification of aggressive tumors. Eur Urol 2010;58:29-30. [http://dx.doi.org/10.1016/j.eururo.2010.05.004]

18. Jentzmik F, Stephan C, Jung K. Reply: Sarcosine in urine after digital examination fails as a marke in prostate cancer detection and identification of aggressive tumors. Eur Urol 2010;58:31-32. [http:// dx.doi.org/10.1016/j.eururo.2010.05.004]

19. Heyns CF, Lecuona AT, Trollip GS. Prostate cancer: prevalence and treatment in African men. J Men's Health and Gender 2005;2(4):400-405. [http://dx.doi.org/10.1016/.jmhg.2005.10.003]

20. Heyns CF, Fisher M, Lecuona A, Van der Merwe A. Prostate cancer among different racial groups in the Western Cape: presenting features and management. S Afr Med J 2011;101(4):267-270.
then 